CERN-TH/2001-253

DCPT/01/78, IPPP/01/39

hep-ph/0109237

\title{
Feynman-diagram evaluation in the electroweak theory with computer algebra
}

\author{
G. Weiglein \\ CERN, TH Division, CH-1211 Geneva 23, Switzerland
}

\begin{abstract}
The evaluation of quantum corrections in the theory of the electroweak and strong interactions via higherorder Feynman diagrams requires complicated and laborious calculations, which however can be structured in a strictly algorithmic way. These calculations are ideally suited for the application of computer algebra systems, and computer algebra has proven to be a very valuable tool in this field already over several decades. It is sketched how computer algebra is presently applied in evaluating the predictions of the electroweak theory with high precision, and some recent results obtained in this way are summarized.
\end{abstract}

\section{INTRODUCTION}

The electroweak and strong interactions of elementary particles are very successfully described by quantized gauge field theories. The quantized nature of these theories manifests itself via corrections beyond the lowest order in the perturbative expansion, which is based on Feynman diagrams. The evaluation of higher-order Feynman diagrams (which are called loop diagrams) is a technically very complicated but on the other hand algorithmic procedure. The development of computer-algebra systems was boosted by the demand for this kind of applications in particle physics, and Schoonschip (1) was one of the first implementations of a powerful computer-algebra program. Further examples of computer-algebra systems that have their roots in particle physics are Reduce (2), Macsyma (3), Mathematica (4), and FORM (5).

Computer-algebra systems allow to perform symbolic manipulations and algebraic calculations without roundoff errors. They are equipped with a number of built-in algorithms which provide the basis for the user to implement his/her own algorithms for handling specific problems. Present-day computer-algebra systems furthermore possess capabilities for communicating with external programs, e.g. with routines for numerical evaluation, text processing and graphics, or with other computer-algebra programs.

Examples of computer-algebra systems being widely used at present in high-energy theory are Mathemat- ica (4), Maple (6) and FORM (5). Mathematica and Maple are general-purpose programs containing a large number of built-in functions (and many additional software packages are available). These programs offer capabilities for both symbolic and numerical computations, support graphical display, and possess user-friendly interactive platforms. The application of these systems to problems in high-energy physics involving expressions with a huge number of terms can be limited by the computing speed or by memory problems. The latter applies in particular to non-local operations, like e.g. factorization, which require to have all terms of an expression available within the physical memory of the computer. FORM, on the other hand, is a program that was specifically optimized for handling very large expressions. It is less user-friendly than Mathematica and Maple, containing much fewer built-in operations and allowing only non-interactive execution. For recent developments concerning the parallelization of FORM, see Ref. (7).

\section{PERTURBATIVE EVALUATION OF GAUGE THEORIES WITH COMPUTER-ALGEBRAIC METHODS}

The concept of treating interactions as a perturbation to a free field theory and performing an expansion in the coupling constants leads to a description of scattering 
processes in terms of Feynman diagrams. The lowestorder prediction, corresponding to the classical limit, for a process with a certain number of external particles (e.g. a $2 \rightarrow 2$ scattering process) is obtained from the sum of the connected diagrams containing the lowest possible power of the coupling constants (which enter via the interaction vertices). These are in general tree-level diagrams. In higher-order diagrams additional interaction vertices give rise to closed loops of propagators, for which an integration over the internal momenta has to be performed.

The prediction for a scattering process of certain fields, assigned to the external legs, and a specified number of loops can be obtained via an algorithmic procedure. In a first step, all topologically different diagrams (for which in renormalizable theories only 3-point and 4-point interaction vertices are possible) have to be generated. Inserting the fields of the model under consideration into the topologies in all possible ways leads to the Feynman diagrams. The Feynman rules translate these graphical representations into mathematical expressions.

Since the loop integrals in general lead to divergences, the expressions need to be regularized (i.e. made mathematically meaningful). In a renormalizable theory the divergences can be absorbed into a redefinition of the parameters of the theory. The renormalization is furthermore necessary in order to fix the physical meaning of the parameters order by order.

The evaluation of the Feynman amplitudes involves a treatment of the Lorentz structure of the amplitude, calculation of Dirac traces etc. At the one-loop level it is possible to reduce all tensor integrals to a set of standard scalar integrals, which can be expressed in terms of known analytic functions. As a consequence, with the existing techniques a wide class of processes with up to four external legs can be evaluated at the one-loop order in massive gauge theories (for a discussion of the technical problems occurring in one-loop processes with six external legs, see e.g. Ref. (8)).

In contrast to the one-loop case, no general algorithm exists so far for the evaluation of two-loop corrections in the electroweak theory. The main obstacle in two-loop calculations in massive gauge theories is the complicated structure of the two-loop integrals, which makes both the tensor integral reduction and the evaluation of scalar integrals very difficult. In general the occurring integrals are not expressible in terms of polylogarithmic functions. For the evaluation of some types of integrals that do not permit an analytic solution numerical methods and expansions in their kinematical variables have been developed.

Applying the appropriate on-shell conditions to the external legs one obtains the S-matrix element from the sum of all contributing Feynman amplitudes. Squaring it and performing the phase space integrations one finally arrives at predictions for cross sections and life times.

Computer-algebraic methods can facilitate most of the above-mentioned steps. Besides benefits from automation, a computer-algebraic treatment is also useful for verifying the correctness of the different steps of a certain calculation. In particular, results obtained at the algebraic level (before inserting specific numerical values for the parameters) are well suited for highly non-trivial checks, e.g. with respect to their UV- and IR-finiteness, gaugeparameter independence, and the validity of SlavnovTaylor identities. As an example, in Ref. (9) a SlavnovTaylor for the two-loop Z-boson self-energy in the electroweak Standard Model (SM) has been verified by showing that the results of about 4000 Feynman diagrams add up to zero algebraically.

As indicated by the above example, powerful computer-algebraic tools are very useful for calculations (in particular of higher-order corrections) in the SM, since the large number of different fields in the SM gives rise to a large number of contributing Feynman diagrams (at the one-loop level typically $O\left(10^{2}\right)$, at the two-loop level $O\left(10^{3}\right)$ ), and the massiveness of the fields makes the evaluation of the loop diagrams very complicated in general. The technical complications are even higher in extensions of the SM. In the Minimal Supersymmetric Standard Model (MSSM) the duplication of the number of fields compared to the SM leads to a plethora of possible interaction vertices and consequently to a large increase in the number of diagrams contributing at a certain order. In QCD, on the other hand, computer-algebraic tools are particularly valuable for multi-loop applications. In Ref. (10), for instance, the four-loop $\beta$ function of QCD has been calculated. This required the computation of about 50000 diagrams, showing clearly the need for a high degree of automation. Similarly, for tree-level processes with many particles in the final state thousands of diagrams can contribute and algebraic methods can be useful for obtaining compact and numerically efficient representations, see e.g. Ref. (11).

Examples of computer-algebra based collections of program packages presently used for higher-order calculations in the electroweak theory and QCD are (where the different programs in each collection mostly use common syntax and can be linked together)

(i) FeynArts (12), FeynCalc (13), FormCalc (14), TwoCalc (9, 15), LoopTools (14), s2lse (16),

(ii) GEFICOM (17), QGRAF (18), MATAD (19), MINCER (20),

(iii) DIANA (21), QGRAF (18), ON-SHELL2 (22),

(iv) xloops (23), GiNaC (24). 
FeynArts, FeynCalc, FormCalc and TwoCalc are written in Mathematica (FormCalc is partially written in FORM). FeynArts is a program for generating all Feynman amplitudes contributing to a certain process to a given order in Mathematica format and for drawing the corresponding Feynman diagrams. As a feature of particular importance for higher-order calculations in the electroweak theory, FeynArts generates not only the unrenormalized diagrams at a given order but also the counterterm contributions at this order and the counterterm diagrams needed for the subloop renormalization. The model files for the electroweak SM (including the Feynman rules for the background-field formulation of the SM (25)) and QCD are predefined in FeynArts. Recently also the model file for the MSSM has been completed (26). In applications to other models, e.g. chiral perturbation theory (27), the appropriate model file has to be provided by the user. This was also the case in previous applications in the two Higgs-doublet model (28) and the $\operatorname{MSSM}(29,31,32,33)$.

FeynCalc and FormCalc are programs (using the FeynArts syntax) for algebraically evaluating one-loop diagrams in the electroweak theory and QCD with up to four external legs in a highly automatized way. FormCalc internally uses an interface to FORM, which is used for the memory- and time-intensive parts of the calculation. FormCalc can directly be linked to LoopTools, which contains routines for the numerical evaluation of scalar one-loop integrals and one-loop tensor coefficients. LoopTools is based on the FF (34) package and provides a Fortran and a $\mathrm{C}++$ library.

As mentioned above, much less tools are available for two-loop calculations in massive gauge theories compared to the one-loop case. The program TwoCalc is based on an algorithm for the tensor reduction of general two-loop 2-point functions, which extends the algorithm for the tensor reduction of one-loop integrals (35). TwoCalc can be used for an automatic reduction of Feynman amplitudes for two-loop self-energies with arbitrary masses, external momenta, and gauge parameters to a set of standard scalar integrals. It can directly be linked to the program s2lse, which is written in $\mathrm{C}++$ and performs the evaluation of the scalar two-loop 2-point integrals by means of one-dimensional integral representations in terms of elementary functions, which allow a fast numerical evaluation with high precision.

The program collection GEFICOM, QGRAF, $M A T A D$, and MINCER is mainly used for calculations in QCD and for the evaluation of QCD corrections to electroweak observables. GEFICOM acts as the master program that calls the other packages. It contains Mathematica and Fortran routines as well as elements written in the script languages $A W K$ and PERL. For details of
GEFICOM and some examples of its applications, see Ref. (17).

The Fortran program QGRAF is an efficient generator for Feynman diagrams. As output the diagrams are encoded in a symbolic notation. Being optimized for high speed, QGRAF is particularly useful for applications involving a very large number (i.e. $O\left(10^{4}\right)$ ) of diagrams. Within GEFICOM, the evaluation of the diagrams proceeds by performing expansions in their kinematical variables. The resulting integrals are then computed with MINCER and MATAD. The program MINCER performs the computation of integrals up to threeloop order where all lines are massless and only one external momentum is non-zero. It makes in particular use of integration-by-parts methods (36). While its original version was written in Schoonschip, the present version of MINCER is realised in FORM. The FORM program MATAD was designed for the computation of vacuum integrals up to three-loop order which contain only one mass scale (i.e. their propagators are either massless or carry a common mass).

The $C$ program DIANA is designed as a master program for higher-order calculations, i.e. it calls the necessary subprograms for a specific computation. It reads the output of QGRAF and can produce a graphical representation for the diagrams if the relevant topologies are pre-defined by the user. For the calculation of the diagrams FORM programs are called, e.g. the package $O N$ SHELL2 which can be used for the calculation of singlescale two-loop 2-point functions (diagrams with only one non-zero mass in the internal lines and the external momentum on the same mass shell).

xloops is a Maple package for calculating certain one-loop and two-loop diagrams in the electroweak SM, which is linked to $\mathrm{C}++$ routines for numerical integration of loop integrals. The symbolic part of xloops is planned to be based in the future on $\mathrm{GiNaC}$, which is a specifically designed framework written in $\mathrm{C}++$.

\section{EXAMPLES OF HIGHER-ORDER RESULTS IN THE SM AND THE MSSM}

In the following some examples are sketched of recent higher-order results obtained with FeynArts, TwoCalc and s2lse in the electroweak SM and the MSSM. Within the SM, higher-order calculations are necessary for the comparison of the theory predictions with the experimental results for electroweak precision observables like $M_{\mathrm{W}}$, $\sin ^{2} \theta_{\text {eff }}$ etc. which have meanwhile reached an accuracy of better than $1 \times 10^{-3}$ (37). The precision tests of the SM allow in particular to set constraints on the mass of the Higgs boson, which is the last missing ingredient of 
the SM and plays a crucial role for a consistent description of massive particles.

In Ref. (39) the currently most accurate prediction for the $\mathrm{W}$-boson mass, $M_{\mathrm{W}}$, within the SM has been obtained. It contains it particular the complete fermionic contributions at the two-loop level, which are treated exactly, i.e. without an expansion in the top-quark or the Higgs-boson mass. The result for $M_{\mathrm{W}}$ is shown in Fig. 1 as a function of the Higgs-boson mass, $M_{\mathrm{H}}$. It is compared with the current experimental value for $M_{\mathrm{W}}$ (37). The present 95\% C.L. lower bound on $M_{\mathrm{H}}$ from the direct search at LEP of $M_{\mathrm{H}}=114.1 \mathrm{GeV}$ (38) is also indicated. The plot shows the well-known preference for a light Higgs boson within the SM. Confronting the theoretical prediction (allowing a variation of the top-quark mass, $m_{\mathrm{t}}$, which at present dominates the theoretical uncertainty, within $1 \sigma$ ) with the $1 \sigma$ region of $M_{\mathrm{W}}^{\exp }$ and the $95 \%$ C.L. lower bound on $M_{\mathrm{H}}$, one finds that at the present level of accuracies the $1 \sigma$ regions do no longer overlap.

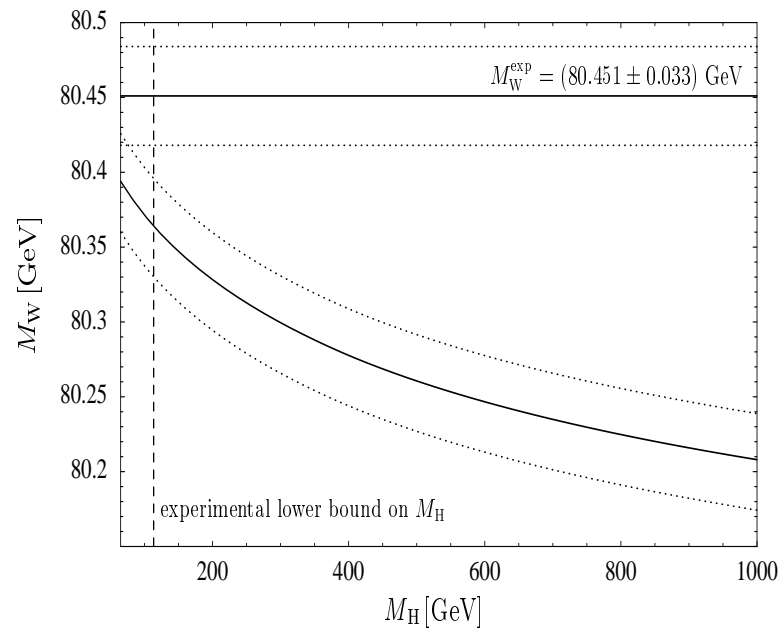

FIGURE 1. The prediction for $M_{\mathrm{W}}$ as a function of $M_{\mathrm{H}}$ for $m_{\mathrm{t}}=174.3 \pm 5.1 \mathrm{GeV}$ is compared with the current experimental value, $M_{\mathrm{W}}^{\exp }=80.451 \pm 0.033 \mathrm{GeV}$ (37), and the experimental $95 \%$ C.L. lower bound on the Higgs-boson mass, $M_{\mathrm{H}}=114.1 \mathrm{GeV}(38)$.

By comparing the SM predictions for the precision observables with those of extended models, it can be investigated whether the data allow a distinction between different kinds of possible models. In Fig. 2 the predictions for $M_{\mathrm{W}}$ in the SM and the MSSM are shown as a function of $m_{\mathrm{t}}$. The MSSM prediction contains the dominant SUSY contributions of $O\left(\alpha \alpha_{s}\right)$ and $O\left(\alpha^{2}\right)$ to the $\rho$ parameter $(29,30)$. The allowed region in the SM corresponds to varying $M_{\mathrm{H}}$ in the interval $114 \mathrm{GeV} \leq M_{\mathrm{H}} \leq 400 \mathrm{GeV}$, while in the region of the MSSM prediction the SUSY parameters are varied, taking into account the constraints from direct searches for SUSY particles. As indicated in the figure, the predictions in the SM and the MSSM give rise to two bands with only a relatively small overlap region. This region corresponds to the SM with a light Higgs, $M_{\mathrm{H}} \lesssim 130 \mathrm{GeV}$, and to the MSSM with heavy superpartners, whose virtual contributions decouple from the electroweak precision observables.

The predictions for $M_{\mathrm{W}}$ in the SM and the MSSM are confronted in Fig. 2 with the current experimental accuracies of $M_{\mathrm{W}}$ and $m_{\mathrm{t}}$ (LEP2/Tevatron, outermost ellipse) and with the prospective accuracies at the LHC and a future Linear Collider (LHC/LC) and at a high-luminosity Linear Collider running in a low-energy mode on the Zboson resonance and the W-pair threshold (GigaZ). As can be read off from the figure, the data on $M_{\mathrm{W}}$ and $m_{\mathrm{t}}$ presently show a slight preference for the MSSM over the SM, which however statistically is not very significant. The figure shows that the next generation of colliders, in particular a Linear Collider in the GigaZ mode, promises an enormous improvement in the experimental accuracies of $M_{\mathrm{W}}$ and $m_{\mathrm{t}}$ (and furthermore also for $\sin ^{2} \theta_{\text {eff }}$ ) which will allow to test the electroweak theory with unprecedented sensitivity (40).

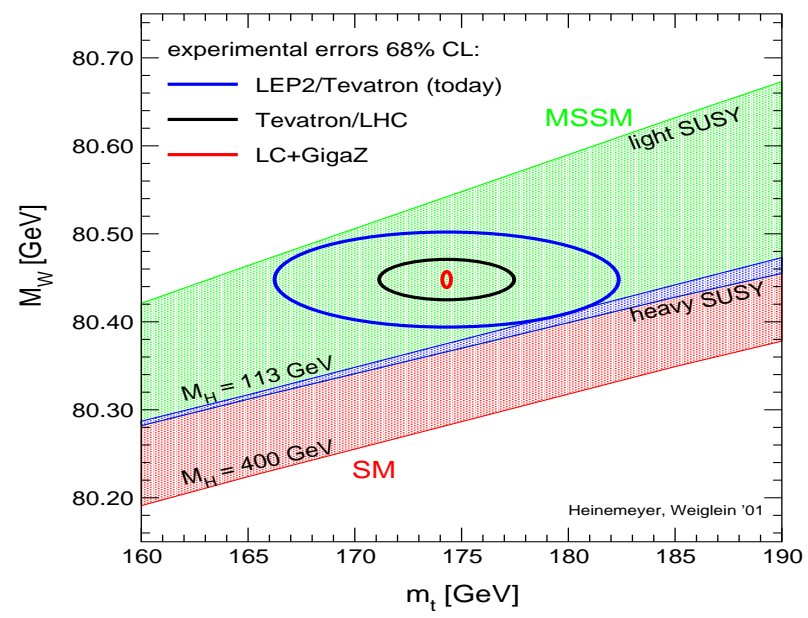

FIGURE 2. The theoretical prediction for $M_{\mathrm{W}}$ within the SM and the MSSM in comparison with the current experimental accuracies (LEP2/Tevatron) and the prospective accuracies at the LHC/LC and at GigaZ.

Besides the indirect constraints from electroweak precision tests, supersymmetric models provide a very stringent direct test since they predict the existence of a relatively light Higgs boson, whose mass can be calculated from the other parameters of the model.

In Ref. (31) a Feynman-diagrammatic result has been obtained for the dominant two-loop contributions to the masses of the neutral $C P$-even Higgs bosons in the MSSM. The algebraic result obtained with FeynArts and TwoCalc has been converted into Fortran code and has been implemented into the program FeynHiggs (41). 
While at the tree-level the lightest $C \mathcal{P}$-even Higgs boson in the MSSM is bounded to be lighter than the Z-boson mass, this bound is shifted upwards to $m_{\mathrm{h}} \lesssim 135 \mathrm{GeV}$ taking into account corrections up to the two-loop order. The highest possible values for $m_{\mathrm{h}}$ are obtained for large values of $\tan \beta$, the ratio of the vacuum expectation values of the two Higgs doublets of the MSSM, large values of the mass of the $C \mathcal{P}$-odd Higgs boson, and a large mixing between the superpartners of the top quark. Comparing the theoretical prediction for the upper bound on $m_{\mathrm{h}}$ as a function of $\tan \beta$ with the experimental exclusion limits obtained at LEP2, it is possible to derive constraints on $\tan \beta$. This is shown in Fig. 3, where the excluded region results from combining the data of the four LEP experiments (42), and the upper (and lower) bound within the MSSM (indicating the boundary to the "theoretically inaccessible" region) has been obtained with FeynHiggs.

The upper plot shows the case of the so-called " $m_{\mathrm{h}}^{\max }$ benchmark scenario" (43), in which the MSSM parameters (for fixed values of $m_{\mathrm{t}}=174.3 \mathrm{GeV}$ and the SUSY scale $M_{\mathrm{SUSY}}=1 \mathrm{TeV}$ ) are chosen such that $m_{\mathrm{h}}$ as a function of $\tan \beta$ takes its maximal values. From the intersection of the experimentally excluded region with the boundary to the theoretically inaccessible region one finds an excluded region of $0.5<\tan \beta<2.4$ within this scenario. In the lower plot the MSSM parameters have been chosen according to the "no-mixing benchmark scenario" (43), which differs from the $m_{\mathrm{h}}^{\max }$ scenario in that vanishing mixing in the scalar top sector has been assumed. In this case a much wider region of $\tan \beta$ values, up to about $\tan \beta \approx 10$, can be excluded for $m_{\mathrm{t}}=174.3 \mathrm{GeV}$ and $M_{\mathrm{SUSY}}=1 \mathrm{TeV}$.

\section{CONCLUSIONS AND OUTLOOK}

The development of powerful computer-algebra systems was triggered by applications in high-energy physics. Computer-algebra tools have extensively been used in this field already for several decades, and many of todays calculations would not have been feasible without computer algebra. A brief overview of computeralgebraic methods for the perturbative evaluation of gauge theories has been given, and some examples have been discussed of recent higher-order results obtained in the electroweak Standard Model and its Minimal Supersymmetric extension.

The use of modern computer-algebra programs goes beyond their application as tools for certain steps of the calculations. As indicated by the above examples of different collections of programs for precision calculations within the theory of the electroweak and strong inter-
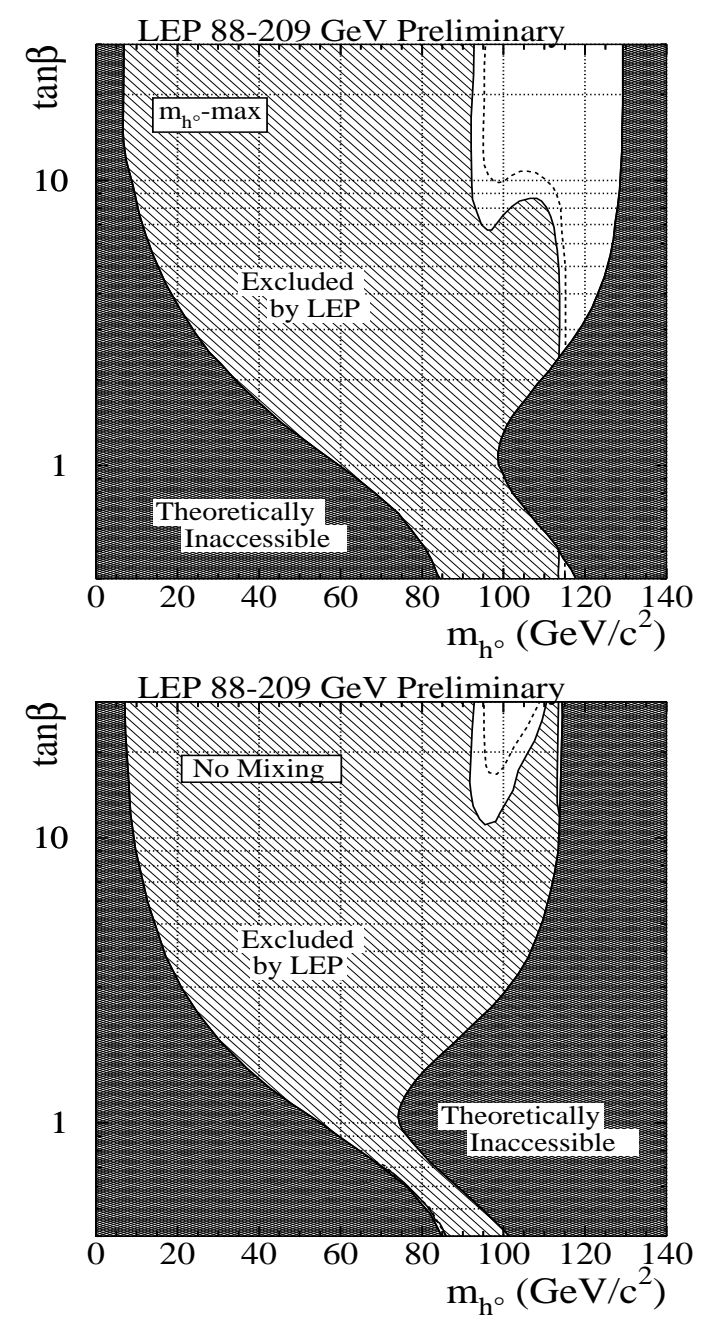

FIGURE 3. The $95 \%$ C.L. bounds on $m_{\mathrm{h}}$ in the $m_{\mathrm{h}}^{\max }$ and the no-mixing benchmark scenarios obtained from combining the data of the four LEP experiments are compared with the upper bound on $m_{\mathrm{h}}$ within the MSSM (42).

actions, an efficient communication of computer-algebra systems with other program components is of particular importance. These external programs can be packages for numerical evaluations, text processing tools, data bases, expert systems, but also other computer-algebra programs being particularly well suited for certain sub-parts of the problem. In order to facilitate this kind of communication, the need for a certain degree of standardization for the integration of program parts and the data transfer between different systems will become more pronounced in the future.

Accordingly, future improvements of soft- and hardware promise a further extension of the applicability of computer-algebra systems in two different ways. On the one hand they could allow highly sophisticated calcula- 
tions which go beyond the scope of present capabilities. On the other hand computer-algebra systems could more and more become parts of general problem-solving environments, where different components are integrated in such a way that the different parts of calculations and the tasks of text processing, graphical representation etc. are handled in the most efficient way.

\section{ACKNOWLEDGMENTS}

The author thanks P. Bhat, the other organizers of ACAT 2000, and the Fermilab Theory Group for the invitation and their kind hospitality during his stay at Fermilab.

\section{REFERENCES}

1. M. Veltman, Schoonschip, CERN preprint, 1967.

2. A.C. Hearn, Reduce User's Manual, (The Rand Corp., Santa Monica, CA, 1985).

3. J. Moses, ACM SIGSAM Bull., 8 (1974) 105.

4. S. Wolfram, Mathematica: A system for Doing Mathematics by Computer, (Addison-Wesley, Reading, Massachusetts, 1988).

5. J.A.M. Vermaseren, Symbolic Manipulation with FORM, (Computer Algebra Netherlands, Amsterdam, 1991);

J.A.M. Vermaseren, these proceedings.

6. B.W. Char, K.O. Geddes, M.W. Gentleman and G.H. Gonnet, in Springer Lecture Notes in Computer Science 162, (Springer, Berlin, 1983), p. 101.

7. D. Fliegner, A. Rétey and J.A.M. Vermaseren, TTP-99-15, hep-ph/9906426; TTP00-13, hep-ph/0007294.

8. A. Vicini, Acta Phys. Pol. B 29 (1998) 2847.

9. G. Weiglein, R. Scharf and M. Böhm, Nucl. Phys. B 416 (1994) 606.

10. T. van Ritbergen, J.A.M. Vermaseren and S.A. Larin, Phys. Lett. B 400 (1997) 379.

11. T. Ohl, these proceedings, IKDA-2000-30, hep$\mathrm{ph} / 0011243$.

12. J. Küblbeck, M. Böhm and A. Denner, Comput. Phys. Commun. 60 (1990) 165;

T. Hahn, KA-TP-23-2000, hep-ph/0012260.

13. R. Mertig, M. Böhm and A. Denner, Comput. Phys. Commun. 64 (1991) 345.

14. T. Hahn, Nucl. Phys. B (Proc. Suppl.) 89B (2000) 231.

15. G. Weiglein, R. Mertig, R. Scharf and M. Böhm, in New Computing Techniques in Physics Research 2, ed. D. PerretGallix (World Scientific, Singapore, 1992), p. 617.
16. S. Bauberger, F.A. Berends, M. Böhm and M. Buza, Nucl. Phys. B 434 (1995) 383;

S. Bauberger, F.A. Berends, M. Böhm, M. Buza and G. Weiglein, Nucl. Phys. B (Proc. Suppl.) 37B (1994) 95; S. Bauberger and M. Böhm, Nucl. Phys. B 445 (1995) 25.

17. R. Harlander and M. Steinhauser, Prog. Part. Nucl. Phys. 43 (1999) 167.

18. P. Nogueira, J. Comput. Phys. 105 (1993) 279.

19. M. Steinhauser, Comput. Phys. Commun. 134 (2001) 335.

20. S.G. Gorishny, S.A. Larin and F.V. Tkachov, INR P-0330 (1984);

S.G. Gorishny, S.A. Larin, L.R. Surguladze and F.V. Tkachov, Comput. Phys. Commun. 55 (1989) 381;

S.A. Larin, F.V. Tkachov and J.A.M. Vermaseren, NIKHEFH/91-18.

21. M. Tentyukov and J. Fleischer, Comput. Phys. Commun. 132 (2000) 124;

J. Fleischer and M. Tentyukov, these proceedings, hep$\mathrm{ph} / 0012189$.

22. J. Fleischer and M.Yu. Kalmykov, Comput. Phys. Commun. 128 (2000) 531.

23. L. Brücher, J. Franzkowski and D. Kreimer, Comput. Phys. Commun. 115 (1998) 140.

24. C. Bauer, A. Frink, R. Kreckel, MZ-TH-00-17, cs/0004015; these proceedings.

25. A. Denner, S. Dittmaier and G. Weiglein, Nucl. Phys. B 440 (1995) 95.

26. T. Hahn and C. Schappacher, KA-TP-18-2001, hep$\mathrm{ph} / 0105349$.

27. U. Bürgi, Nucl. Phys. B 479 (1996) 392.

28. W. Beenakker, A. Denner and A. Kraft, Nucl. Phys. B 410 (1993) 219.

29. A. Djouadi, P. Gambino, S. Heinemeyer, W. Hollik, C. Jünger and G. Weiglein, Phys. Rev. Lett. 78 (1997) 3626; Phys. Rev. D 57 (1998) 4179.

30. S. Heinemeyer and G. Weiglein, BNL-HET-01-7, hep$\mathrm{ph} / 0102317$.

31. S. Heinemeyer, W. Hollik, and G. Weiglein, Phys. Rev. D 58 (1998) 091701; Phys. Lett. B 440 (1998) 296; Eur. Phys. Jour. C 9 (1999) 343.

32. A. Arhrib and G. Moultaka, Nucl. Phys. B 558 (1999) 3.

33. S. Heinemeyer, these proceedings, BNL-HET-00-46, hep$\mathrm{ph} / 0102318$.

34. G.J. van Oldenborgh and J.A.M. Vermaseren, Z. Phys. C 46 (1990) 425;

G.J. van Oldenborgh, NIKHEF-H/90-15.

35. G. Passarino and M. Veltman, Nucl. Phys. B 160 (1979) 151.

36. F.V. Tkachov, Phys. Lett. B 100 (1981) 65;

K.G. Chetyrkin and F.V. Tkachov, Nucl. Phys. B 192 (1981) 159. 
37. J. Drees, talk given at Lepton Photon 01, Rome, July 2001, to appear in the proceedings.

38. ALEPH, DELPHI, L3 and OPAL Collaborations, The LEP working group for Higgs boson searches, CERN-EP/2001055, hep-ex/0107029.

39. A. Freitas, W. Hollik, W. Walter and G. Weiglein, Phys. Lett. B 495 (2000) 338;

A. Freitas, S. Heinemeyer, W. Hollik, W. Walter and G. Weiglein, Nucl. Phys. B (Proc. Suppl.) 89B (2000) 82; CERNTH/2001-018, hep-ph/0101260.

40. J. Erler, S. Heinemeyer, W. Hollik, G. Weiglein and P.M. Zerwas, Phys. Lett. B 486 (2000) 125;

S. Heinemeyer and G. Weiglein, hep-ph/0012364.

41. S. Heinemeyer, W. Hollik and G. Weiglein, Comput. Phys. Commun. 124 (2000) 76; hep-ph/0002213.

42. ALEPH, DELPHI, L3 and OPAL Collaborations, The LEP working group for Higgs boson searches, LHWG Note 2001-04, hep-ex/0107030.

43. M. Carena, S. Heinemeyer, C. Wagner and G. Weiglein, CERN-TH/99-374, hep-ph/9912223. 